\title{
Status of the H1 Very Forward Proton Spectrometer
}

\author{
Laurent Favart * \\ I.I.H.E., Université Libre de Bruxelles, CP 230 \\ 1050 Brussels - Belgium \\ on behalf of the H1 Collaboration
}

\begin{abstract}
The Very Forward Proton Spectrometer (VFPS) of the H1 experiment at HERA is collecting data since 2005. The fiber detectors in the Roman pots located at 218 and $222 \mathrm{~m}$ downstream from the $\mathrm{H} 1$ interaction point, tag and measure diffractively scattered protons with a high acceptance in the $x_{\mathbb{P}}$ range [0.01, 0.025]. The experimental set up and the spectrometer tagging performance using diffractive events collected during 2006 and 2007 are discussed.
\end{abstract}

\section{Introduction}

In recent years considerable progress has been achieved in the partonic interpretations of diffractive processes in $e-p$ collisions (see e.g. [2]), Most of diffractive studies performed up to now at HERA have been based on the characteristic presence of a rapidity gap in the diffractive final state. The precision of this method is limited by the uncertainty related to the presence of dissociated proton background events. The only precise and unambiguous way of studying diffraction is by tagging the diffracted proton and measuring its four momentum by means of a proton spectrometer. Such devices have been used by the H1 and ZEUS Collaborations and have delivered interesting results, but their acceptances are small, with the result that the collected statistics are limited and large systematic errors affect the measurements. To fully profit from the HERA luminosity upgrade in the study of diffraction after the year 2003, a Very Forward Proton Spectrometer (VFPS) which identifies and measures the momentum of the diffracted proton with a high acceptance has therefore been installed by H1. This contribution reports the VFPS tagging performance using diffractive events collected during HERA running at high energy (27.5 GeV for the electron/positron beam and $920 \mathrm{GeV}$ for the proton) in 2006 and 2007.

\section{Roman Pot detectors}

The VFPS [3] is a set of two "Roman pots" located at $118 \mathrm{~m}$ and $222 \mathrm{~m}$ downstream of the H1 interaction point. Each pot consists of an insert into the beam pipe, allowing two tracking detectors equipped with scintillating fibres to be moved very close to the proton beam.

Many aspects of the design of the Roman pots, including the stainless plunger vessel and the scintillating fiber detectors, are adaptations of the FPS proton spectrometer [4], installed and operational in $\mathrm{H} 1$ since 1994. Both detectors of each Roman pot consists of two planes of scintillating fibres oriented at $\pm 45^{\circ}$ w.r.t. the horizontal plane and moving perpendicularly to the beam line direction. Each detector allows to reconstruct of the position of one impact point of the scattered proton trajectory with a precision of about $100 \mu \mathrm{m}$. For triggering

*This work is supported by the Fonds National de la Recherche Scientifique Belge (FNRS). 
purposes each detector is sandwiched between 2 scintillating planes which are connected to different PM's. A trigger signal, corresponding to a activity in at least 3 planes out of four, is delivered separately for each station at the first trigger level.

\section{$3 \quad$ VFPS installation and running}

The VFPS have been installed at the very end of 2003. Radiation damage of the optical readout fiber prohibited data taking during 2004. Hence data available for physics analysis started in 2005. The bulk of data were taken in 2006 and 2007, they correspond to and integrated luminosity of $140 p b^{-1}$. From an operational point of view, the VFPS was into data taking position for $70 \%$ of the luminosity collected by H1.

The $p$ beam orbit has been modified in April 2006 to increase the VFPS acceptance. A large fraction of protons with a energy loss above $2 \%$ w.r.t. the beam energy are hitting the beam pipe around $200 \mathrm{~m}$ when the nominal orbit is used. The orbit has been changed moving the $p$ outwards HERA by $6 \mathrm{~mm}$ at about $200 \mathrm{~m}$ from the interaction point. This procedure leads to an

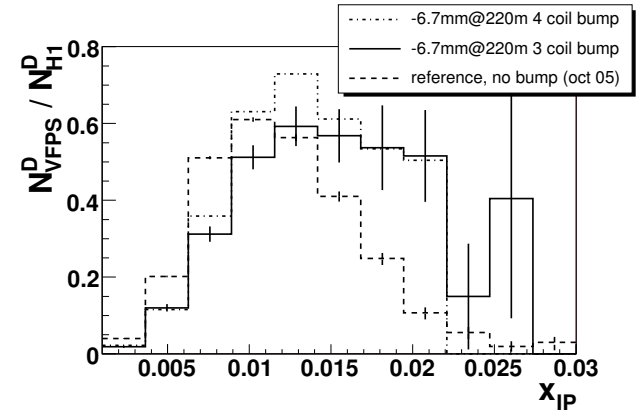

Figure 1: Ratio of events tagged by the VFPS to the diffractive events seen in $\mathrm{H} 1$ (see section 4) as a function of $x_{\mathbb{P}}$ for different beam optics. increase of the rate of events tagged by the VFPS at $x_{\mathbb{P}}>0.015$ (see Fig. 1).

\section{Inclusive Diffraction in DIS regime}

To study the description of the beam optics and of the VFPS system by our simulation, first, VFPS tagged events, i.e. with a fired trigger, are compared to the full sample of diffractive events selected using the information from the main detector using the rapidity gap method (see e.g. [5]). The full event sample is selected asking for an electromagnetic cluster in the backward (lepton beam direction) calorimeter SpaCal of more than $10 \mathrm{GeV}$ (corresponding to the scattered electron candidate), a reconstructed vertex and that the most forward particle in the main detector has pseudo-rapidity of less than 2.5 (this latest condition is equivalent to asking for a rapidity gap). Additionally the Forward Muon Detector should not have recorded a signal above the noise level. One can then look to what fraction of this sample largely dominated by diffractive events is tagged by VFPS. This selection was applied to produce the Fig. 1 discussed in the previous section. Over the $140 p b^{-1}$ collected, 880,000 events are tagged by the VFPS. If a kinematic cut of $Q^{2}>10 \mathrm{GeV}^{2}$ is applied, 215,000 events remain.

This sample is compared to the sum of diffractive and background contributions as estimated by Monte Carlo. In Fig. 2 data corresponding to about 1 month of running in $e^{+} p$ 
mode with a $6 \mathrm{~mm}$ bump applied $\left(24 p b^{-1}\right)$ are compared to Monte Carlo predictions (see figure caption for details).
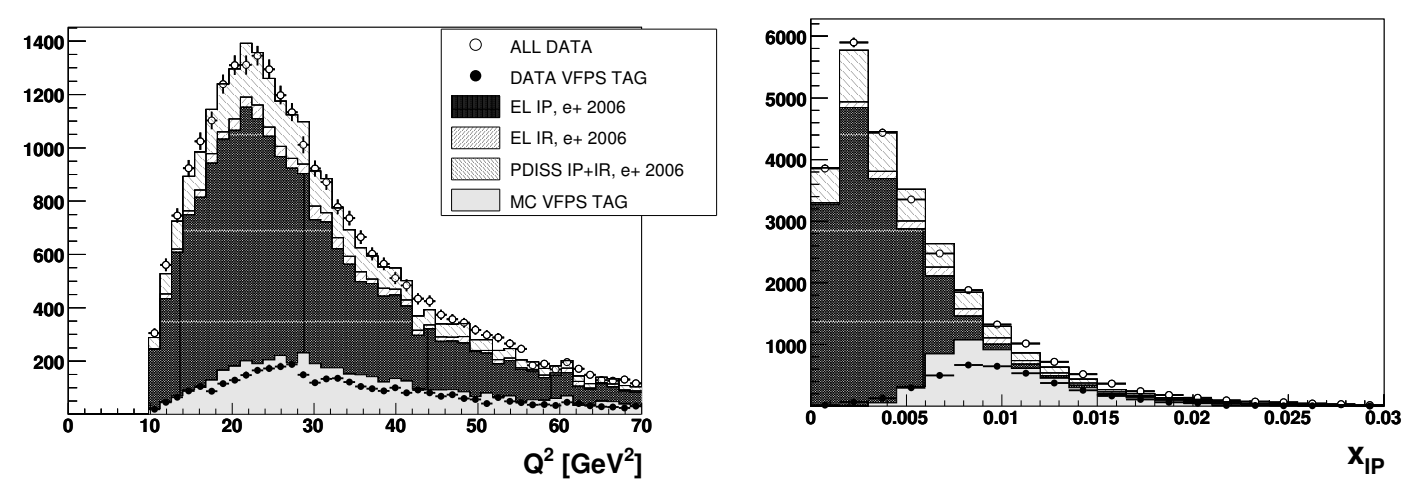

Figure 2: Diffractive events in DIS (empty points) are compared to VFPS tagged events (full points) and to Monte Carlo simulation. Left: as a function of $Q^{2}$. Right: as a function of $x_{\mathbb{P}}$. The Monte Carlo simulation contains contributions from Pomeron exchange with elastically scattered proton (EP IP), Reggeon exchange with elastically scattered proton (EP IR), Pomeron and Reggeon exchange with proton dissociation (PDISS IP+IR). Among them simulated events tagged by the VFPS are shown (MC VFPS TAG).

A good agreement is found between the full data sample and the Monte Carlo (normalized to the data luminosity). The trend in $x_{\mathbb{P}}$ of VFPS tagged sample is described by the Monte Carlo. A more precise understanding of the VFPS acceptance is needed and will lead to a better description in $x_{\mathbb{P}}$.

\section{Diffractive dijets}

\section{Diffractive dijets in DIS}

The analysis of diffractive dijets in DIS regime is based on $42.6 e^{-}$and $54.7 e^{+} p b^{-1}$ taken in 2006. Additionally to the selection applied in the previous section, a requirement of at least two jets (using the $K t$ algorithm) is asked, with a minimal transverse momentum in the photon-proton frame of $p_{T, 1}^{*}>5.5 \mathrm{GeV}$ and $p_{T, 2}^{*}>4 \mathrm{GeV}$ respectively for the first and the second jet. The jets are asked to be well contained in the main detector, by requirering $\eta_{j 1, j 2} \in[-1,2]$.

The $x_{\mathbb{P}}$ distribution is shown in Fig. 3a comparing the full dijet sample and VFPS tagged dijet sample. This plot illustrates the well suited acceptance of the VFPS for the dijets production in diffraction. In Fig. 3b the transverse momentum of the first jet in the laboratory frame is shown. Here again the full dijet sample is compared to the VFPS tagged dijet sample.

\section{Diffractive dijets in photoproduction}

To record with a high efficiency diffractive dijet events in photoproduction tagged by the VFPS, a special trigger has been developed. It allows to lower the threshold in jet transverse 

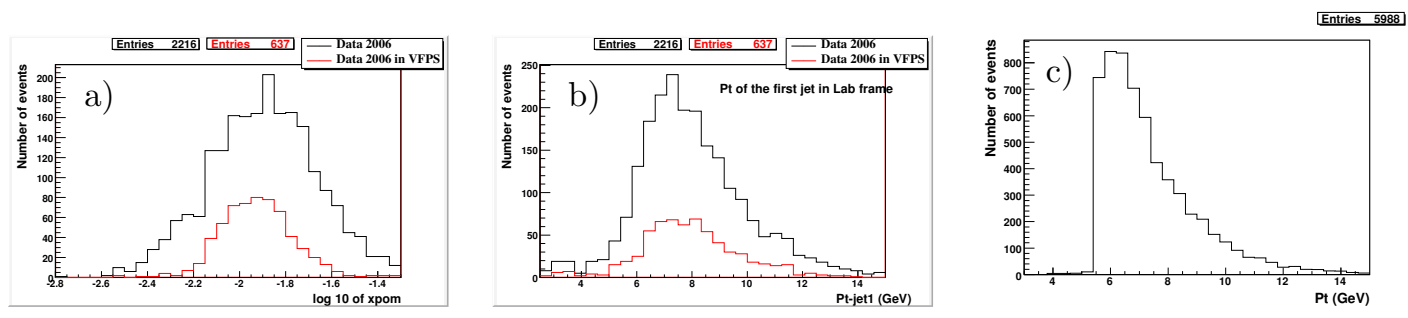

Figure 3: a) $x_{\mathbb{P}}$ distribution of dijet diffractive events in DIS. The highest histogram corresponds to the full dijet sample and the lowest one to the VFPS tagged dijet sample. b) Highest transverse momentum of jets in the laboratory frame of dijet diffractive events in DIS. The highest histogram corresponds to the full dijet sample and the lowest one to the VFPS tagged dijet sample. c) Highest transverse momentum of jets in the laboratory frame of dijet VFPS tagged diffractive events in photoproduction.

momentum down to $5 \mathrm{GeV}$. A luminosity of $23.7 p b^{-1}$ has been collected in 2006 and 2007 with that trigger corresponding to a selected sample of 6000 events. The selection criteria are the same as in the dijet DIS case except that the scattered electron escapes undetected, at small angle, in the beam pipe. Figure 3c shows the transverse momentum of the first jet in the laboratory frame (equivalent to the photon-proton frame for the transverse direction in the present photoproduction case). The distribution of VFPS tagged events cannot be compared to a full dijet sample as no trigger allowed to keep efficiently those events down to a transverse momentum of $5 \mathrm{GeV}$.

\section{Conclusion}

The VFPS has run successfully collecting a luminosity of $140 \mathrm{pb}^{-1}$. The observed acceptance is high (above 60\%) in a region of $x_{\mathbb{P}}$ around $10^{-2}$. The trend in $x_{\mathbb{P}}$ of diffractive events tagged by the VFPS is described by the Monte Carlo simulation. Important statistics have been collected (880,000 diffractive DIS events, 800 dijets diffractive DIS events and 6000 dijets diffractive events in photoproduction) for diffractive structure function measurement and QCD factorisation tests. The proton momentum reconstruction based on VFPS fiber information is still in progress.

\section{References}

[1] Slides: http://indico.cern. ch/contributionDisplay.py? contribId=96\&sessionId=7\&conf Id=9499

[2] L. Favart, Experimental review of diffractive phenomena, in proceedings of the $10^{\text {th }}$ International Baryons Conference (BARYONS 2004), [hep-ex/0501052].

[3] Proposal for Installation of a Very Forward Proton Spectrometer in H1 after 2000, PRC-01/00, H1 note H1-05/00-582, http://www-h1.desy.de/h1det/tracker/vfps/

[4] Upgrade of the H1 Forward Spectrometer, PRC-96/01

[5] J. Lukasik, these proceedings. 